written extensively about Gödel's contribution to Einstein's theory of relativity. Yourgrau and Goldstein both write about the friendship that developed between these two very different scholars. Gödel was a shy, reclusive man with a tendency to paranoia, whereas Einstein was worldly and outgoing. Nevertheless, the two were seen each day walking together to and from their offices at the Institute for Advanced Study in Princeton, obviously enjoying one another's company.

The equations of general relativity, having superseded Newton's account of gravitation, provide one of the great successes of twentieth-century physics. In 1949, Gödel discovered unexpected solutions to these equations corresponding to universes in which no universal temporal ordering is possible. A hypothetical inhabitant of such a universe could, in principle, travel to his own past. Yourgrau argues that because time fails to exist in these Gödel universes, and because the differences between such universes and our own are accidental, time can't exist in our world either. I doubt that many readers would be convinced by this argument.

When his wife was hospitalized, Gödel literally starved himself to death, unwilling to eat anything not prepared by her. Referring to his sad end, Goldstein makes the untoward suggestion that he might have imagined that he was living in an actual Gödel universe in which he could look forward to an eternal recurrence, reliving his life over and over again. A staunch believer in an afterlife, Gödel would hardly have sought such a fate. In any case, weird as the Gödel universes are, such eternal recurrence is not one of their properties.

Martin Davis is professor emeritus, New York University, and is a visiting scholar at the University of California, Berkeley. He can be contacted at 3360 Dwight Way, Berkeley, California 94704-2523, USA.

\section{The dark side of astronomy}

Empire of the Stars: Friendship, Obsession and Betrayal in the Quest for Black Holes

by Arthur I. Miller

Little, Brown/Houghton Mifflin: 2005.

416 pp. $116.99 / \$ 26$

\section{Thanu Padmanabhan}

Science, unfortunately, is done by humans. This makes scientific debates subject to the usual follies of human interaction, prejudices and lack of objectivity. One classic example in the modern history of astrophysics is the dispute between Arthur Eddington and Subrahmanyan Chandrasekhar (also known as Chandra) regarding the fate of stars with masses above a critical value. In 1930, at the age of just 19, Chandra made the pioneering discovery that such objects should continue to collapse to a singularity, a mathematical point of infinite density. Eddington, the leading authority in astrophysics at the time, ridiculed this conclusion. And thereby hangs a tale.

The fate of such massive objects is an extremely important question in astrophysics. Chandra was predicting the existence of black holes, although this idea was years away from reaching maturity. $\mathrm{He}$ reached his viewpoint by combining the laws of special relativity with those of quantum theory as applied to particles such as electrons when they are in a state in which they are said to be relativistically degenerate. Chandra's calculations, related to the pressure of the relativistically degenerate gas, led him to the conclusion that gravity will inexorably crush a sufficiently massive body to a point.

If Eddington had merely expressed discomfort at matter collapsing to a point-like singularity of infinite density, that would have been fair enough. Even today we do not understand what actually happens to matter that falls into a black hole, and those

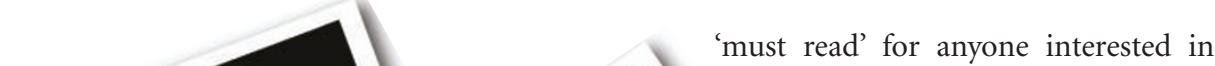
astrophysics or the history of science. The book begins with the dramatic moment when Chandra gave a seminar on his results at a meeting of the Royal Astronomical Society in London in January 1935, and describes how Eddington made mincemeat of it. It then goes backwards in time, caricaturing both of the dramatis personae, Chandra and Eddington. Having put the pieces together, Miller goes on to describe how this particular episode left its mark on Chandra. As well as the story of Chandra's encounter with Eddington, Miller tells of the progress made in this subject over the years, and an appendix

of us who have thought uncomfortable with the notion of a singularity. But Eddington questioned Chandra's logic and his calculation, and raised irrelevant objections. Later work completely vindicated Chandra, but

the early humiliation left a deep scar. We can only speculate about what would have happened if Eddington had taken the scientifically correct stand and helped to develop Chandra's ideas further.

Historian Arthur Miller tells this story in a lively and exciting narrative in Empire of the Stars. The book is so beautifully written that I read it in one sitting. The scientific issues, as well as the personalities of everyone involved in the debate, have been described in picturesque detail without either mincing words or making unsubstantiated claims. Wherever necessary, Miller is careful to give references to the sources from which he has gathered the information, making the work fairly authoritative. In short, this book is a 
these underhand methods, but what can one do?" Similarly, Miller points out that Chandra continued to complain bitterly that leading physicists never supported him against Eddington, despite the fact that Paul Dirac, Rudolf Peierls and Maurice Price wrote an important paper that backed him up.

On the sidewalks of the narration, one also picks up some interesting snippets, such as how the Nobel laureate Raman declared that there will be no astrophysicists within miles of Bangalore; or how Chandra decided to spend some time in America because of the "underhanded dealings going on in Indian scientific circles"; and why Robert Oppenheimer had a successful collaboration with Richard Tolman.

In summary, this is an entertaining and illuminating book about a key issue in contemporary astrophysics. The author is to be congratulated on producing an authoritative description in a manner that is so thoroughly enthralling.

Thanu Padmanabhan is at Pune University, Ganeshkhind, Pune 411 007, India.

\section{Destroying the zombic hunch}

\section{Sweet Dreams: Philosophical \\ Obstacles to a Science of \\ Consciousness}

by Daniel C. Dennett

MIT Press: 2005. 216 pp, \$28, £18.95

\section{Susan Blackmore}

Has the devil lost his horns? Dan Dennett has been demonized because of his tough materialist stance on consciousness and his claim that we are conscious machines with nobody at home inside. But now he updates his theory and, I think, presents a softer version of it.

Sweet Dreams is a collection of essays and lectures written between 1999 and 2005 in which Dennett tries to freeze time and present a 'best' version of his evolving ideas. There are problems with this, especially as some chunks are somewhat disconcertingly repeated from one chapter to the next, but the overall picture gives a good idea of where Dennett's thinking has been going.

He describes his task as being to explain away qualia-based intuitive objections to materialism, and this he does using some of his favourite examples. One is the everpopular philosopher's zombie, an imaginary creature who looks, acts and speaks like a normal person but has no subjective

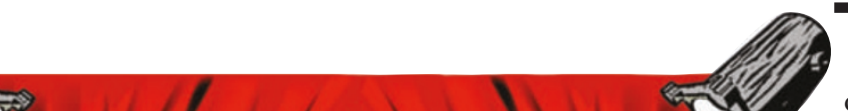

watching the magic show of conscious experiences from the safety of the cartesian theatre. Dennett contrasts those theorists to whom it is obvious that a theory that leaves out the subject cannot explain consciousness, with those to whom it is obvious that the subject has to vanish. The first type must be wrong, he says: "A good theory of consciousness should make a conscious mind look like an abandoned factory."

All these ideas will be familiar to lovers and haters of Dennett, as will his attacks on Mary the colour-scientist (who knows all there is to know about colour perception but lives in a black-and-white world) and the idea of first-person science. But he does add some new twists and, as ever, delights with his quirky turns of phrase. I laughed out loud at his example of a folk theorem as ludicrous as some of the ones that people claim are obvious: "If you burp, sneeze, and fart all at the same time, you die." But let's have some evidence, he says.

What may be less familiar is Dennett's updating of his theory of consciousness, from the original 'multiple drafts' theory, through the delightfully named 'fantasy echo', to his latest ideas of 'fame in the brain' or 'cerebral celebrity'.

In a paper originally published as an overview for a special 2001 issue of Cognition, he joins in the experience or qualia. It's easy to imagine a zombie — or at least to think you are imagining one - says Dennett, and he calls this "falling for the zombic hunch", which traps people into believing that consciousness is separate from brain function. Dennett has tried to murder the zombie before, explaining how people fail to follow the rules when they think they are imagining one, but now with the concept of the zombic hunch he explores the damage done by this false intuition. Don't worry, he says, if you are patient and open minded it will pass, or mutate into a less virulent form. Just as we still feel as though the Earth stands still, in the future people may still feel the zombic hunch, but they won't believe it. They will know that mechanistic theories of consciousness do the whole job, so we don't need the concept of qualia.

Demystifying consciousness is Dennett's forte, and is probably the main reason for his status as the devil - after all, people like mysteries. Some even think that if consciousness is explained they will be diminished as people, being turned into mere 'things'. They don't like the idea that there is no one at home inside their head, no audience "gathering consensus" that favours 'global workspace' theory. Originally proposed by Bernard Baars in A Cognitive Theory of Consciousness (Cambridge University Press, 1988), this theory is explicitly based on a theatre metaphor in which the contents of consciousness are illuminated by a spotlight of attention, shining on the stage of working memory. The idea is that many specialist brain systems contribute information to the global workspace; its contents are then broadcast to the rest of the system and this global availability is experienced as a conscious state.

Doesn't this go against everything Dennett has been fighting for? He tries to explain why not. Consciousness, like fame, is not an intrinsic property of brain processes but is more like political influence or clout; conscious events are the ones that have widespread effects in the brain. So we must not think that becoming famous in the brain magically ignites the glow of conscious qualia or lets pictures into the cartesian theatre to be watched by the conscious subject; the effects are enough. Fame in the brain does not lead to consciousness - it is consciousness. 